\title{
Rol de voriconazol y caspofungina en terapia antifúngica
}

\author{
TERESA BIDART H.
}

\section{Voriconazol and caspofungin in antifungal therapy}

Palabras claves: Voriconazol; Caspofungina; Candidiasis; Aspergilosis. Key word: Voriconazole; Caspofungin; Candidiasis; Aspergillosis.

\section{Introducción}

Las infecciones fúngicas invasoras (IFI) son una importante causa de morbilidad y mortalidad en pacientes inmunocomprometidos. El número de pacientes expuestos al riesgo de IFI ha aumentado sustancialmente en las ultimas dos décadas en relación a la aparición de la epidemia del SIDA, uso de quimioterapia intensiva en pacientes oncohematológicos, uso de fármacos antirechazo en pacientes receptores de trasplante y la mayor utilización de dispositivos intravasculares.

Las IFI se asocian con una mortalidad inaceptable sobre $40 \%$ en infecciones del torrente sanguíneo causadas por Candida albicans y mayor de $50 \%$ en aspergilosis invasoras. Varios factores, incluyendo la dificultad en diagnosticar micosis profundas, el empeoramiento de la función inmune de los pacientes y la alta tasa de falla terapéutica, contribuyen al pobre pronóstico de las IFI.

Recientes estudios epidemiológicos han confirmado el incremento de la importancia de infecciones causadas por especies de hongos resistentes, particularmente Candida sp resistentes a fluconazol y hongos que previamente eran considerados meros colonizantes o contaminantes, llamados hongos emergentes, algunos de los cuales son resistentes a todos los antifúngicos y que causan IFI graves; hongos filamentosos tales como Scedosporium prolificans o Fusarium sp y algunos zigomicetos causan infecciones letales que no responden a las terapias actuales.

$\mathrm{La}$ resistencia primaria a anfotericina $\mathrm{B}$ ha emergido en paralelo con el incremento de IFI causadas por hongos emergentes tales como
Trichosporon beigelii, Candida lusitaniae o Candida guilliermondii, hongos filamentosos del grupo de hialohiphomicosis tales como Fusarium $\mathrm{sp}$, Scopulariosis sp y Scedosporium sp y ciertos hongos dematiaceos. Muchos de estos hongos muestran resistencia primaria o intrínseca a anfotericina B y pueden causar infecciones invasoras asociadas con alta mortalidad.

Las infecciones invasorivas por Candida sp, Aspergillus sp y otros hongos filamentosos han incrementado en pacientes que viven bajo profunda inmunosupresión.

Las opciones de tratamiento antifúngico han sido limitadas, hasta la reciente emergencia de nuevas formulaciones de anfotericina $\mathrm{B}$ y de itraconazol sumado a los nuevos triazoles de amplio espectro actualmente en estudio (posaconazol) o aprobados recientemente como voriconazol, y las equinocandinas, una nueva clase de antifúngicos de las cuales el primer agente aprobado es caspofungina, ahora disponible en Chile.

La realización de ensayos clínicos con fármacos antifúngicos es difícil por la heterogeneidad de los pacientes y la dificultad para establecer un diagnóstico definitivo.

Pero la toxicidad de anfotericina B, las bajas tasas de curación de las IFI y la aparición de hongos emergentes y resistentes ha estimulado el desarrollo de nuevos antifúngicos, aumentado el armamento terapéutico.

El uso de nuevas formulaciones de anfotericina $\mathrm{B}$ a fines de 1990 y la introducción de los azoles a fines de 1980 representaron un importante avance en el manejo de infecciones fúngicas por su seguridad y perfil farmacocinético. La introducción de estos nuevos agentes antifúngicos facilitó

Clínica Santa María, Santiago, Chile. 
Tabla 1. Clasificación general de antifúngicos

\begin{tabular}{ll}
\hline Antibióticos & Polienos: anfotericina, nistatina y natamicina \\
& No polienos: griseofulvina \\
Azzoles & Imidazoles: miconazol \\
& Triazoles: ketoconazol, itraconazol, fluconazol, voriconazol, posaconazol, ravuconazol \\
& Tópicos: bifonazol, butoconazol, clotrimazol, econazol, fenticonazol, sulconazol, \\
& tioconazol, terconazol \\
Pirimidinas fluoradas & Flucitosina \\
Equinocandinas & Caspofungina, micafungina y anidulafungina \\
Alilaminas & Terbinafina y naftifina \\
Otros & Yoduro potásico, ciclopirox, tolnaftato \\
\hline
\end{tabular}

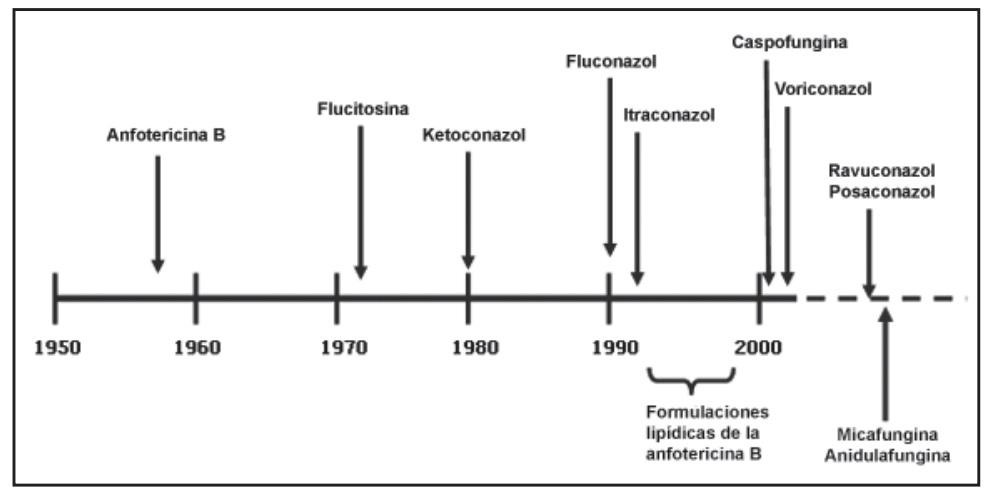

Figura 1. Historia de los antifúngicos.

un abordaje más agresivo en la profilaxis y tratamiento de infecciones fúngicas en la década pasada pero favoreció la emergencia de organismos resistentes. La presión de selección debido a la continua exposición a azoles parece tener un rol esencial en el desarrollo de resistencia en Candida sp.

\section{Voriconazol}

Es un triazol de segunda generación de amplio espectro derivado sintético del fluconazol. Inhibe el citocromo P450 dependiente de 14 lanosterol demetilasa, la enzima requerida para la síntesis de

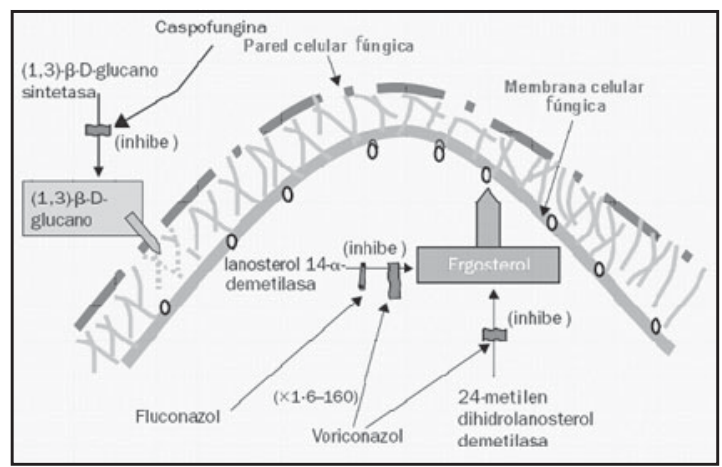

Figura 2. Mecanismos de acción de los diferentes antifúngicos.

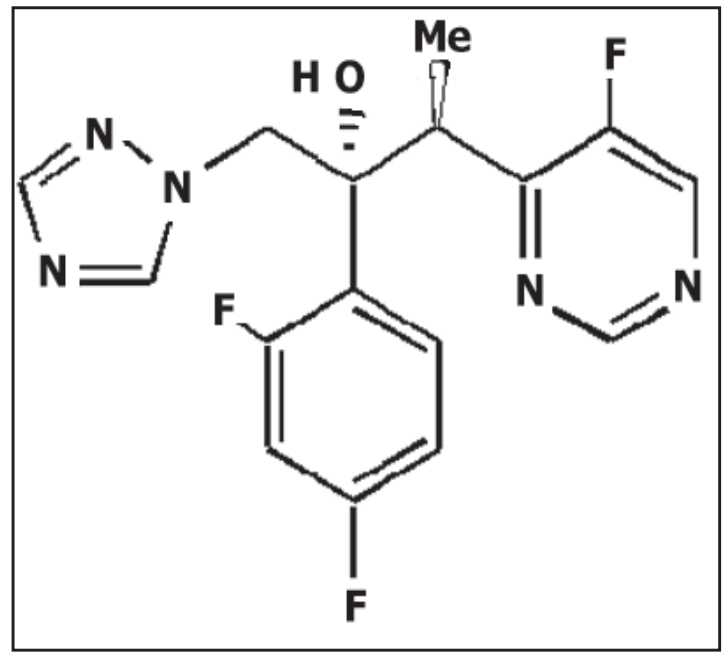

Figura 3. Estructura química de voriconazol.

ergosterol. Esta enzima es importante en la producción y mantención de la pared celular fúngica. Voriconazol es mucho más potente inhibidor de la enzima fúngica que fluconazol, resultando en un amplio espectro antifúngico.

Voriconazol es fungistático contra Candida $s p$, siendo activo frente a Candida, incluyendo las especies $C$. glabrata y C. krusei que son resistentes a fluconazol, aunque frente a éstas las CIM son mayores que para otras especies de Candida. 
Tabla 2. Clasificación general de los antifúngicos

\begin{tabular}{llll}
\hline Agente & $\begin{array}{l}\text { Sitio de acción en } \\
\text { la célula fúngica }\end{array}$ & Actividad & Implicancias clínicas \\
\hline Anfotericina B & Membrana & $\begin{array}{l}\text { Unión a ergosterol causa muerte } \\
\text { celular }\end{array}$ & $\begin{array}{l}\text { Potente actividad fungicida de } \\
\text { amplio espectro }\end{array}$ \\
Azoles & Membrana & $\begin{array}{l}\text { Inhibe el citocromo p450 respon- } \\
\text { sable de la síntesis de ergodterol; } \\
\text { daño de membrana citoplasmática }\end{array}$ & $\begin{array}{l}\text { Actividad fungistática de poten- } \\
\text { cia y espectro variable }\end{array}$ \\
Equinocandinas & Pared & $\begin{array}{l}\text { Inhibe la síntesis de glucano; dis- } \\
\text { rupción de la estructura de la pared } \\
\text { celular causando lisis de la célula }\end{array}$ & $\begin{array}{l}\text { Actividad antifúngica potente } \\
\text { de amplio espectro. Potencial } \\
\text { para efecto aditivo en terapia } \\
\text { combinada }\end{array}$ \\
\hline
\end{tabular}

Adaptado de Maertens J Int Infect Dis 2003; 7: 94-101.

Tiene actividad fungicida contra la mayoría de las especies de Aspergillus incluyendo A. terreus. También tiene una buena actividad contra hongos dismórficos (Coccidioides sp, Histoplasma sp, Blastomyces $\mathrm{sp}$, etc). Casos reportados in vitro sugieren buena actividad contra Penicillium sp, Fusarium sp, Scedosporium sp y Phaeohyphomicosis pero no es activo frente a Zygomycetes sp.

Farmacocinética - farmacodinámica. Voriconazol oral posee una excelente biodisponibilidad con farmacocinética similar a voriconazol intravenoso cuando se administra una hora ${ }^{3,4}$ antes o una hora después de las comidas. Se metaboliza en el hígado vía citocromo P-450 y sus metabolitos carecen de actividad antifúngica. El estado de equilibrio se alcanza tras 5 ó 6 días de tratamiento pero si se administra una dosis de carga de $6 \mathrm{mg} / \mathrm{kg}$ cada 12 horas este estado se podría alcanzar el primer día. Sus concentraciones en LCR son aproximadamente $50 \%$ de las plasmáticas. Es necesario ajustar dosis en pacientes con deterioro moderado de la función hepática y su empleo debería ser sopesado en pacientes con insuficiencia hepática grave. La formulación oral no precisa ajuste de dosis en presencia de insuficiencia renal, pero la administración intravenosa debería ser considerada sólo en pacientes con clearence de creatinina mayor a $50 \mathrm{ml} / \mathrm{min}$ ya que ocurre acumulación del excipiente que acompaña a voriconazol intravenoso (sulfobutileter - $\beta$ ciclodextrina).

En adultos, voriconazol exhibe una farmacocinética no lineal por lo cual hay una substancial variabilidad interindividual en las concentraciones, no así en lo niños en quienes la eliminación sí es lineal.

Efectos adversos. Es un fármaco generalmente bien tolerado. El efecto adverso más frecuente es un trastorno reversible de la visión, observado hasta en $30 \%$ de los pacientes. El segundo más

\begin{tabular}{|llllll|}
\hline Candida albicans & AnF & Flu & Itra & Vori & Casp \\
Candida tropicalis & & & & & \\
Candida parapsilosis & & & & & \\
Candida krusei & & & & \\
Candida glabrata & & & \\
Criptococcus neoformans & & & \\
Histoplasma capsulatum & & \\
Blastomyces dermatitis & & & \\
Coccidiodes immitis & & & \\
Paracoccidiodes brasiliensis & $?$ & & \\
Aspergillus fumigatus & $?$ & & \\
Zygomycetes spp & & & \\
Fusarium solani & $?$ & \\
Scedosporium apiospermum & $?$ & \\
\hline
\end{tabular}

Figura 4. Espectro de acción de los diferentes antifúngicos. La intensidad del color gris indica la intensidad del efecto antifúngico. 
observado es el exantema cutáneo (8\%), no debiendo olvidarse su potencial de fotosensibilidad. Produce elevación de las enzimas hepáticas (10 $15 \%$ ) reversible al discontinuar el medicamento, con mayor frecuencia que fluconazol, describiéndose algunos casos de hepatitis grave. Debido a su metabolización a través del citocromo P450 el potencial de interacciones farmacológicas es elevado (Tabla 3 ).

\section{Uso clínico de voriconazol}

\section{Infecciones por Aspergillus sp}

La eficacia de voriconazol en aspergilosis invasora ha sido confirmada en un estudio abierto no comparativo que lo evaluó como fármaco de primera línea o bien, frente al fracaso de otros antifúngicos. Cuando se administró como tratamiento inicial se obtuvo una respuesta favorable (completa o parcial) en 59\% de los casos y en $38 \%$ de los pacientes que habían fracasado con otro tratamiento antifúngico. Posteriormente Herbecht y cols compararon anfotericina B versus voriconazol en terapia primaria de arpergilosis invasora en 277 pacientes. A las 12 semanas de iniciar el tratamiento antifúngico se observó una respuesta favorable en $53 \%$ de los pacientes tratados con voriconazol versus $32 \%$ de los tratados con anfotericina $\mathrm{B}$, reduciendo la mortalidad en 71 versus $58 \%$ respectivamente.

\section{Infecciones por Pseudallescheria sp, Scedosporium sp y Fusarium sp}

Voriconazol fue aprobado para tratamiento de rescate en infecciones por Pseudallescheria boydii y su forma asexuada $S$. apiospermum sp y Fusarium sp en pacientes intolerantes o refractarios a otros agentes antifúngicos. Perfect y cols observaron una respuesta favorable en $30 \%$ de las infecciones causadas por Scedosporium sp y en $46 \%$ de las infecciones por Fusarium sp al administrar voriconazol tras el fracaso o toxicidad grave de otros antifúngicos.

\section{Usos no licenciados}

Voriconazol ha demostrado ser tan eficaz como fluconazol en el tratamiento de la esofagitis por Candida sp en pacientes con SIDA. Perfect y cols evidenciaron una respuesta favorable en $58 \%$ de los pacientes con infección refractaria por Candida sp.

Tabla 3. Principales interacciones medicamentosas de voriconazol

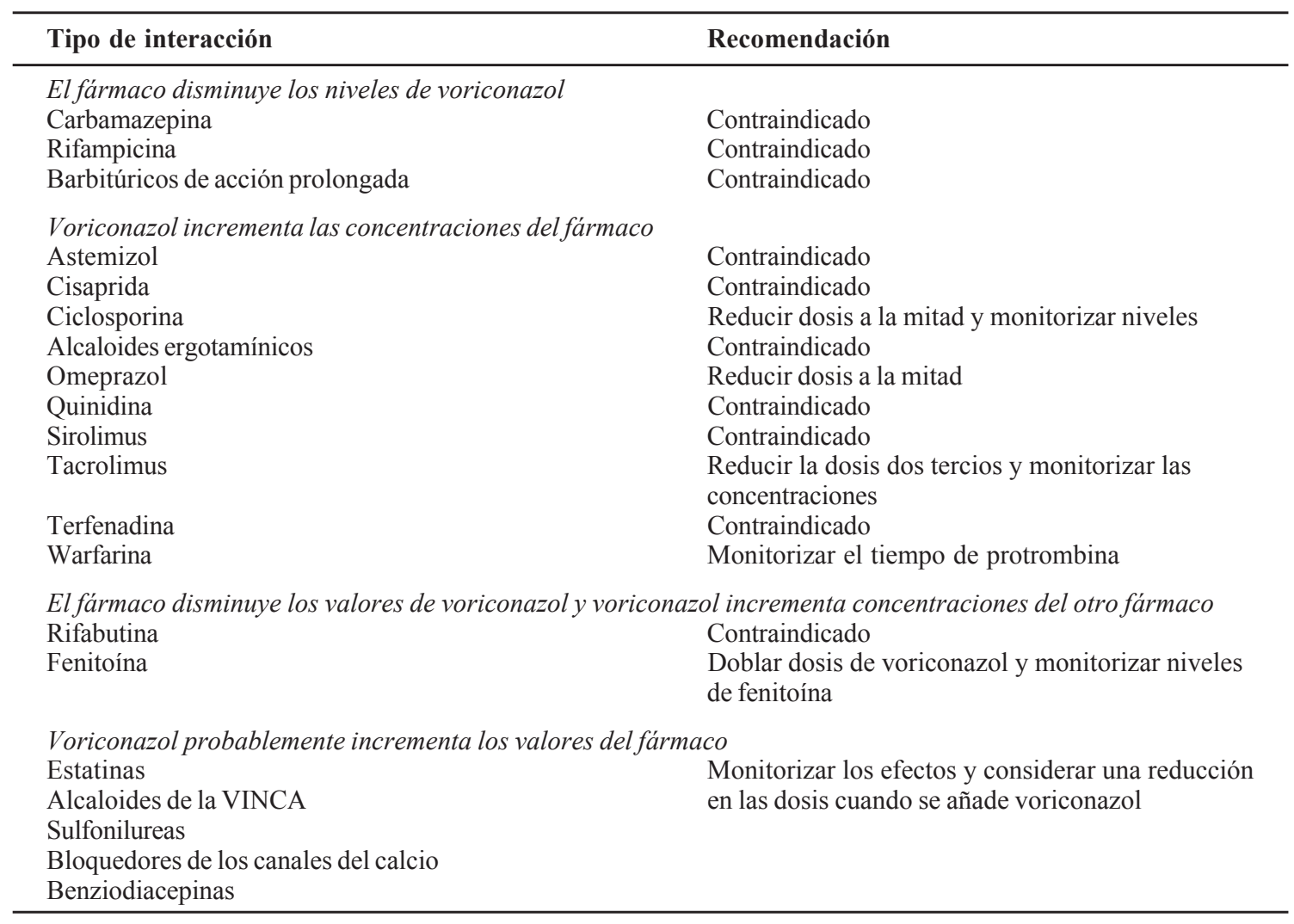

Adaptado de ref. 4 
No existe evidencia clara sobre la eficacia de voriconazol en el tratamiento de candidemia o candidiasis invasora.

\section{Criptococosis, histoplasmosis, blastomicosis}

Voriconazol demostró excelente actividad in vitro contra Criptococcus neoformans y alcanza buenos niveles en LCR. Pero faltan estudios clínicos que evalúen la eficacia tanto en criptococosis como en micosis endémicas para aprobar su recomendación.

\section{Uso empírico de voriconazol en neutropenia febril}

Los resultados de un gran estudio multicéntrico randomizado que comparó voriconazol $(\mathrm{n}=415)$ con anfotericina B para tratamiento empírico de pacientes con neutropenia febril son controversiales.

\section{Resumen de recomendaciones de voriconazol}

- Aprobado en aspergilosis invasora.

- Aprobado en terapia de rescate de infecciones por Fusarium sp y Scedosporium sp.

- Aprobado en candidiasis esofágica refractaria a fluconazol .

- Su rol en candidemia y otros hongos no está definido.

- La utilidad de su uso empírico en neutropenia febril es controversial.

- No es activo contra Zygomycetes.

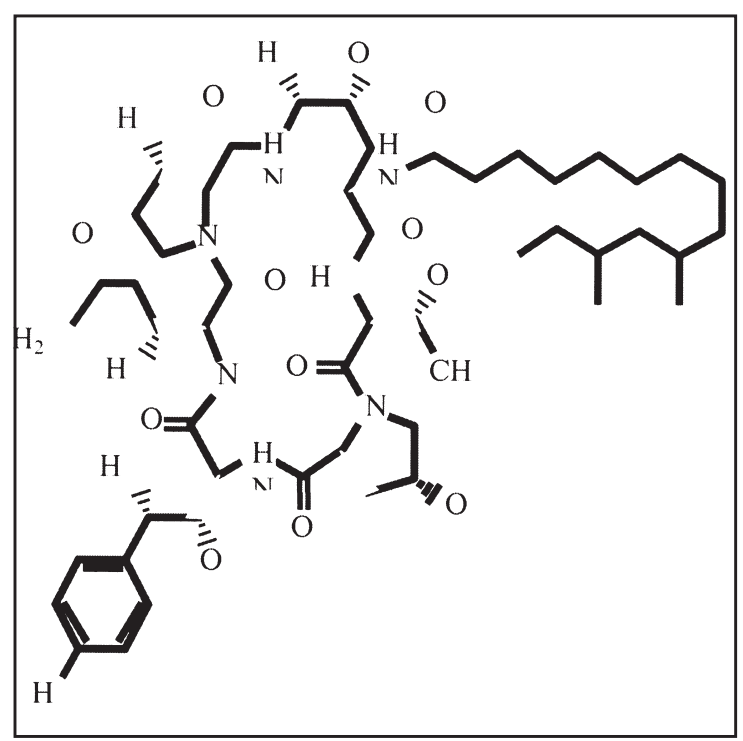

Figura 5. Estructura química de caspofungina.

\section{Caspofungina}

Es el primer representante de una nueva clase antifúngicos denominados equinocandinas que poseen un nuevo mecanismo de acción: interfieren en la síntesis de la pared del hongo.

Mecanismo de acción. Inhibición de las síntesis de $\beta 1,3 \mathrm{D}$ glucano componente clave de la pared de la célula fúngica, produciendo inestabilidad osmótica y muerte celular. El 1,3 $\beta$-D glucano no está presente en las células de los mamíferos lo que explica la escasa toxicidad del fármaco en humanos

No existe un método de referencia para valorar las CIM de caspofungina frente a levaduras y hongos filamentosos; por lo tanto, hay que ser cautelosos en la extrapolación de los datos in vitro a la actividad in vivo. Su espectro antifúngico está restringido a Candida sp y Aspergillus sp Caspofungina es fungicida in vitro e in vivo contra la mayoría de Candida sp y fungistática contra Aspergillus sp. No ha sido demostrada resistencia cruzada con polienos y azoles. No presenta actividad in vitro frente a C. neoformans, Trichosporom beigelii ni frente a hongos de la familia Dematiaceae, Rhizopus o Fusarium sp. Es activa in vitro y en forma experimental contra la forma quística de Pneumocystis jiroveci.

Farmacodinamia y efectos adversos. $\mathrm{La}$ biodisponibilidad oral de caspofungina es pobre, sólo existe formulación intravenosa. Tiene una vía media de 9 a 11 horas y un clearence renal mínimo, no se requiere ajuste de dosis en presencia de falla renal y no es dializable. En pacientes con insuficiencia hepática moderada o grave (Child $>7$ ) debe reducirse la dosis a $35 \mathrm{mg} /$ día. Posee pocos efectos adversos entre los que destacan: fiebre, cefalea, flebitis, rash y elevación transitoria de enzimas hepáticas $(10 \%)$.

Interacciones medicamentosas. La farmacocinética de caspofungina es alterada por la coadministración de ciclosporina o rifampicina $(35 \%$ de incremento del área bajo la curva y $30 \%$ de reducción, respectivamente). La coadministración de caspofungina y efavirenz, nevirapina, fenitoína, dexametasona o carbamazepina puede traducirse en reducciones clínicamente significativas de la concentración de caspofungina, por lo que se debe aumentar la dosis de mantención del antifúngico a $70 \mathrm{mg} /$ día. Mientras que el área bajo la curva de tacrolimus es reducida en 20 a $25 \%$, por lo tanto es necesario monitorizar sus niveles. 


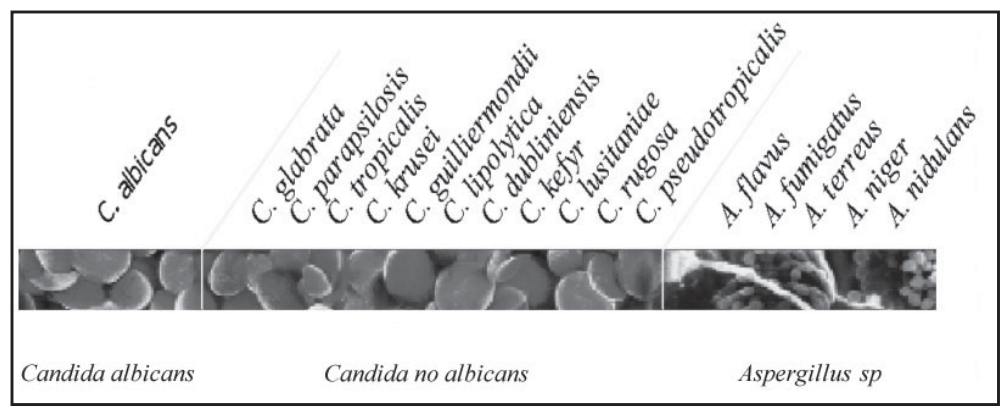

Figura 6. Espectro de acción de caspofungina.

\section{Eficacia clínica}

\section{Infecciones por Candida sp}

Caspofungina ha demostrado eficacia comparable a la de anfotericina $\mathrm{B}$ y fluconazol en candidiasis orofaríngea y esofágica y en el tratamiento de candidiasis esofágica refractaria a fluconazol. Entre los estudios en pacientes con infección sistémica por Candida destaca el de Mora-Duarte y cols. Este ensayo randomizó pacientes con candidiasis invasora ( $83 \%$ tenía candidemia y $10 \%$ peritonitis) a recibir el caspofungina $(70 \mathrm{mg}$ al día $1 \mathrm{y}$ luego $50 \mathrm{mg} /$ día $)$ o anfotericina $\mathrm{B}$ convencional $(0,6-1,0 \mathrm{mg} / \mathrm{kg}$ día $)$ por 14 días. Se consiguió éxito terapéutico en $73 \%$ de los pacientes tratados con anfotericina B $(\mathrm{p}=0,08)$. Si sólo se analizaban los pacientes que habían recibido $\geq 5$ días de terapia las cifras de curación aumentaban a 85 y $65 \%$ respectivamente $(p=0,03)$. La toxicidad de caspofungina fue muy inferior a la observada con anfotericina $\mathrm{B}$.

\section{Infecciones por Aspergillus sp}

Caspofungina ha sido aprobada para terapia de rescate en adultos con aspergilosis invasora refractaria a tratamiento antifúngico convencional o quienes son intolerantes a estas terapias. Maertens y cols evaluaron 56 pacientes refractarios o intolerantes a otras terapias observando una respuesta favorable (completa o parcial) en $41 \%$ de los pacientes que habían recibido a lo menos una dosis de caspofungina, frente al $4 \%$ en los refractarios a terapia previa $(n=46)$ y $70 \%(n=10)$ en intolerantes a terapia previa.

\section{Terapias combinadas}

La disponibilidad de antifúngicos con distintos mecanismos de acción han puesto en marcha el estudio de asociaciones de antifúngicos y/o citoquinas, esperando mejorar la sobrevida de infecciones fúngicas invasoras en el futuro.

\section{Resumen de caspofungina}

- Es una nueva clase de antifúngicos que interfiere en la síntesis de la pared celular.

- Produce menos interacciones farmacológicas.

- Aprobado para el tratamiento de candidiasis.

- Aprobado para terapia de rescate en aspergilosis.

\section{Uso de nuevos antifúngicos en profilaxis}

El uso de equinocandinas es una excelente opción para profilaxis antifúngica considerando su perfil de seguridad, larga vida media espectro de acción contra levaduras y la mayoría de las especies de Aspergillus y su mínima interacción con otras fármacodrogas. Su principal limitante es la no existencia de formulación oral.

El uso de voriconazol en profilaxis también está siendo evaluado, considerando su amplio espectro y su disponibilidad en formulación oral pero ya están emergiendo zigomicosis invasoras en relación a su uso.

\section{Conclusiones}

Las terapias antifúngicas son cada vez más complejas debido a las interacciones farmacológicas, toxicidad y aparición de resistencia a los antifúngicos clásicos.

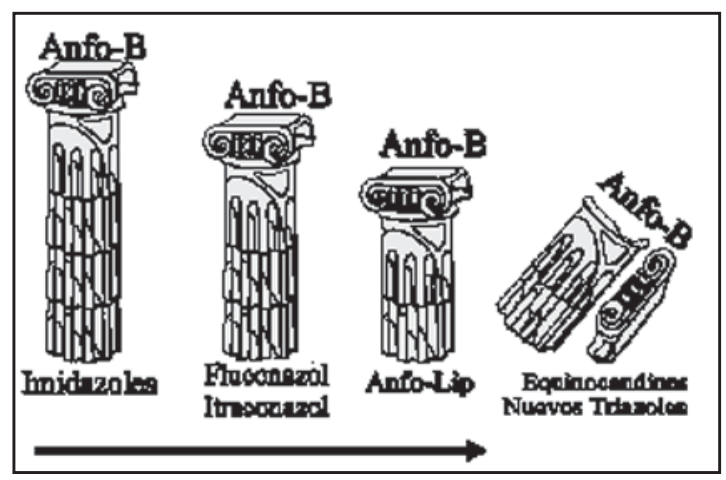

Figura 7. Espectro de acción. 
Existen nuevas alternativas de antifúngicos con espectro de acción más amplio y menos tóxicos.

\section{Bibliografía}

1.- Herbrecht R, Denning D W, Patterson T F, Bennett J E, Greene R E, Oestmann J W, et al Voriconazole versus amphotericin $\mathrm{B}$ for primary therapy of invasive aspergillosis. N Engl J Med 2002; 347: 408-15.

2.- Masia Canuto M, Gutiérrez Rodero T. Antifungal drug resistance to azoles and polyenes. Lancet Infect Dis 2002; 2: 550-63.

3.- Johnson L B, Kauffman C A. Voriconazole: a new triazole antifungal agent. Clin Infect Dis 2003; 36: 630-7.

4.- Lumbreras C, Lizasoain M, Aguado J M. Sistemic antifungal agents. Enferm Infecc Microbiol Clin 2003; 21: $366-79$.

5.- Perfect J R, Marr K A, Walsh T J, Greenberg R N, DuPont B, de la Torre-Cisneros $\mathrm{J}$ et al. Voriconazole treatment for less-common, emerging, or refractory fungal infections. Clin Infect Dis 2003; 36: 1122-31.

6.- Rubin Z A, Somani J. New options for the treatment of invasive fungal infection. Semin Oncol 2004; 31 (Suppl 4): 91-8.

7.- Zeng J, Kamei K, Zheng Y, Nishimura K. Susceptibility of Pseudallescheria boydii and Scedosporium apiospermum to new antifungal agents. Jpn J Med Mycol 2004; 45: 101-4.

8.- Ally R, Schurmann D, Kreisel W, Carosi G, AguirreBengoa K, Dupont B, et al. A randomized, doubleblind, double dummy, multicenter trial of voriconaloze andl fluconazole in the treatment of esophageal candidiasis in imnmunocompromised patients. Clin Infect Dis 2001; 33: 1447-54.

9.- Perea S, Patterson T F. Antifungal resistance in pathogenic fungi. Clin Infect Dis 2002; 35: 1073-80.

10.- Hamza N S, Ghannoum M A, Lazarus H M. Choices aplenty: antifungal prophlylaxis in hematopoietic stem cell transplant recipients. Bone Marrow Transplantation 2004; 1-13.

11.- Siwek G T, Dodgson K J, Magalhaes-Silverman M, Bartelt L A, Kilborn S B, Hoth P L et al. Invasive zygomycosis in hematopoietic stem cell transplant recipients receiving voriconazole prophylaxis. Clin Infect Dis 2004; 39: 584-7.

12.- van Duin D, Cleare W, Zaragoza O, Casadevall A, Nosanchuk J D. Effects of voriconazole on Criptococcus neoformans. Antimicrob Agents Chemother 2004; 48: 2014-20.

13.- Keating G, Figgitt D. Caspofungin: a review of its use in oesophageal candidiasis, invasive candidiasis and invasive aspergillosis. Drugs 2003; 63: 2235-63.

14.- Denning D W. Echinocandin antifungal drugs. Lancet 2003; 362: 1142-51.

15.- Mora-Duarte J, Betts R, Rotstein C, Colombo A L, Thompson-Moya L, Smietana J et al. Comparison of caspofungin and amphotericin B for invasive candidiasis. N Engl J Med 2002; 347: 2020-9.

16.- Maertens J, Boogaerts M. Caspofungin in the treatment of candidosis and aspergillosis. Int $\mathrm{J}$ Infect Dis 2003; 7: 94-101.

17.- Deresinski S C, Stevens D A. Caspofungin. Clin Infect Dis 2003; 36: 1445-57.

18.- Nucci M, Marr K A, Queiroz-Telles F, Martins C A, Trabasso P, Costa S, et al. Fusarium infection in hematopoietic stem cell transplant recipients. Clin Infect Dis 2004; 38: 1237-42.

19.- Steinbach W J, Schell W A, Blankenship J R, Onyewu C, Heitman J, Perfect J R. In vitro interactions between antifungals and immunosuppressants against Aspergillus fumigatus. Antimicrob Agents Chemother 2004; 48: 1664-9.

20.- Garbino J, Rohner P, Kolarova L, Ondrusova A, Lew D. Successful treatment of pulmonary invasive aspergillosis with voriconazol in patients who failed conventional therapy. Infection 2003; 31: 241-3.

21.- Perea S, González G, Fothergill A W, Kirkpatrick W $\mathrm{R}$, Rinaldi M G, Patterson T F. In vitro interaction of caspofungin acetate with voriconazole against clinical isolates of Aspergillus sp. Antimicrob Agents Chemother 2002; 46: 3039-41

22.- Kontoyiannis D P, Hachem R, Lewis R E, Rivero G A, Torres H A, et al. Efficacy and toxicity of caspofungin in combination with liposomal amphotericin B. As primary or salvage treatment of invasive aspergillosis in patients with hematologic malignances. Cancer 2003; 98: 292-9.

23.- Manavathu E K, Alangaden G J, Chandraskar P H. Differential activity of triazoles in two-drug combinations with the echinocandin caspofungin against Aspergillus fumigatus. J Antimicrob Chemother 2003; 51: 1423-5.

24.- Hossain M A, Reyes G H, Long L A, Mukherjee P K, Ghannoum M A. Efficacy of caspofungin combined with amphotericin B against azole-resistance Candida albicans. J Antimicrob Chemother 2003; 51: 1427-9.

25.- Walsh T J, Pappas P, Winston D J, Lazarus H, Peterson F, Raffalli J et al. Voriconazol compared with liposomal amphotericin B for empirical antifungal therapy in patients with neutropenia and persistent fever. N Engl J Med 2002; 346: 225-34.

26.- Walsh T J, Karlsson M O, Driscoll T, Arguedas A G, Adamson P, Sáez Llorens X, et al. Pharmacokinetics and safety of intravenous voriconazole in children after single or multiple-dose administration. Antimicrob Agents Chemother 2004; 48: 2166-72.

Correspondencia a:

Teresa Bidart Hernández

tbidart@yahoo.es 